\title{
Densidad, estructura poblacional, reproducción y supervivencia de cuatro especies de plantas útiles en el Valle de Tehuacán, México
}

\author{
Density, population structure, reproduction and survival of four useful plat species in the \\ Tehuacán Valley, Mexico
}

\author{
Héctor Godínez-Alvarez, Miguel Jiménez, Marleth Mendoza, Fabiola Pérez, Patricia Roldán, Leticia Ríos- \\ Casanova y Rafael Lira* \\ Unidad de Biología, Tecnología y Prototipos (UBIPRO), Facultad de Estudios Superiores Iztacala, Universidad Nacional Autónoma de México. Avenida \\ de los Barrios 1, Los Reyes Iztacala, Tlalnepantla 54090, Apartado postal 314, Estado de México, México. \\ *Correspondencia: rlira@servidor.unam.mx
}

\begin{abstract}
Resumen. En este trabajo se analizó densidad, estructura poblacional, reproducción y supervivencia individual de 4 especies de plantas útiles con el fin de determinar el estado en el que se encuentran sus poblaciones y capacidad de regeneración, así como la importancia relativa de la reproducción sexual y la propagación vegetativa para el reclutamiento de 2 de estas especies. Las poblaciones de todas las especies tienen densidades variables y están compuestas por plantas de tamaño intermedio. Las plantas de tamaño pequeño y grande son escasas. La producción de frutos varió entre las poblaciones de cada especie, aunque no se observaron diferencias en el número de semillas por fruto y la proporción de semillas germinadas. La supervivencia de las plántulas fue baja y dependió de las condiciones ambientales. La propagación vegetativa es el mecanismo más común para el reclutamiento de las especies que presentan esta forma de reproducción. Se discuten los posibles factores físicos, biológicos y humanos que podrían limitar la capacidad de regeneración de las poblaciones y su efecto sobre el aprovechamiento de estas plantas.
\end{abstract}

Palabras clave: Agave marmorata, cactus, Castela tortuosa, densidad, estructura poblacional, germinación, mezquite, Prosopis laevigata, reclutamiento, Stenocereus stellatus, supervivencia.

\begin{abstract}
This work analyzes density, population structure, individual reproduction and survival of 4 useful plant species, in order to determine the current status of their populations and their regeneration capacity. The relative importance of sexual reproduction and vegetative propagation in the recruitment of 2 of these species was also analyzed. Populations of all species are composed of medium size plants and their density is variable. Small and large plants are scarce. For all species fruit production varied between populations, although there were no significant differences in the number of seeds per fruit and proportion of germinated seeds. Seedling survival was low and affected by environmental conditions. Vegetative propagation was the most common mechanism for the recruitment of those species with this type of reproduction. The potential physical, biological, and human factors that might limit the population regeneration capacity, as well as their effects on the use of these plants are discussed.
\end{abstract}

Key words: Agave marmorata, cacti, Castela tortuosa, density, germination, mesquite, population structure, Prosopis laevigata, recruitment, Stenocereus stellatus, survival.

\section{Introducción}

El Valle de Tehuacán, una zona árida ubicada en el límite de los estados de Puebla y Oaxaca, posee una gran diversidad biológica y cultural. En esta zona existen alrededor de 2800 especies de plantas, 184 especies de animales vertebrados y habitan 7 grupos humanos (Dávila et al., 2002). Las plantas útiles representan un elemento importante de la diversidad biológica, ya que se han

Recibido: 10 octubre 2007; aceptado: 28 abril 2008 registrado más de 900 especies (Pérez-Negrón y Casas, 2007). Estas plantas proporcionan recursos naturales como forraje, medicina, alimento, combustible y materiales de construcción, por lo que son fundamentales para la existencia de los grupos humanos de la región.

De acuerdo con Pérez-Negrón y Casas (2007), alrededor de 60 especies de plantas útiles están sujetas a distintos tipos de manejo in situ, como la tolerancia, el fomento y la protección. La recolección de productos en el campo es otra práctica frecuente que permite a los pobladores obtener diversos productos, como alimento, 
medicina y leña. El uso directo de los productos obtenidos de las plantas y la comercialización de algunos de ellos complementan la economía de los pobladores (Casas et al., 2001; Pérez-Negrón y Casas, 2007).

Debido a que la subsistencia de los grupos humanos depende en cierta medida de las plantas útiles, la determinación de algunos parámetros demográficos de las poblaciones de estas plantas y su capacidad de regeneración contribuiría a garantizar el aprovechamiento de los recursos naturales. Distintos atributos demográficos como la densidad, la estructura poblacional, la producción de flores, frutos y semillas, y el establecimiento de los individuos pueden proporcionar la información necesaria para realizar estas evaluaciones. Así por ejemplo, la estructura poblacional permite hacer reflexiones respecto a la viabilidad de una población (Elzinga et al., 2001). Las poblaciones con una proporción alta de individuos seniles y una baja proporción de individuos reproductivos o de plántulas, potencialmente pueden decrecer. Por su parte, el análisis de la producción de flores, frutos y semillas, así como el del establecimiento de los individuos permite conocer algunos de los factores que limitan la reproducción y la supervivencia individual y que afectan la capacidad de regeneración de las poblaciones (Elzinga et al., 2001).

En la actualidad pocos trabajos han realizado mediciones de algunos atributos demográficos, no obstante su importancia para conocer el estado en que se encuentran las poblaciones de las plantas útiles (Camargo-Ricalde et al., 2004; Godínez-Alvarez et al., 2005; Mendoza, 2006; Pérez, 2004; Roldán, 2004). La mayoría de trabajos realizados hasta el momento han estado enfocados en el inventario de las plantas útiles, sus usos y formas de manejo (Avendaño et al., 2006; Blanckaert et al., 2004; Carmona y Casas, 2005; Casas et al., 2001), así como al análisis de los sistemas reproductivos, la variación morfológica y la diversidad genética de especies particulares de plantas (Cruz y Casas, 2002; Casas et al., 1997, 1999a, 1999b; Otero-Arnaiz et al., 2003).

En este trabajo se evalúan los atributos demográficos de Stenocereus stellatus (Pfeiffer) Riccob. (Cactaceae), Prosopis laevigata (Humb. y Bonpl. ex Willd.) M.C. Johnston (Leguminosae), Castela tortuosa Liebm. (Simaroubaceae) y Agave marmorata Roezl. (Agavaceae), 4 especies importantes de plantas de uso múltiple en el Valle de Tehuacán (Cuadro 1). Para cada especie, se evalúa la densidad, la estructura poblacional, la producción de frutos y semillas, la germinación en condiciones controladas y la supervivencia de las plántulas en el campo. Stenocereus stellatus y A. marmorata se reproducen sexual y vegetativamente, por lo que para estas especies también se analiza la importancia relativa de estos modos de reproducción en el establecimiento de nuevos individuos. Consideramos que la medición de estos atributos es relevante ya que permite evaluar rápida y fácilmente las poblaciones de varias especies de plantas útiles y proporciona información para el diseño de planes de seguimiento y proyectos particulares de ciertas especies. El análisis de las poblaciones con otras herramientas, como los modelos matriciales, permite conocer detalladamente el efecto de ciertas etapas del ciclo de vida sobre la tasa de crecimiento poblacional. Sin embargo, el uso de estas herramientas no es siempre factible, sobretodo cuando el tiempo y los recursos económicos son limitados, por lo que son necesarias otras alternativas para la evaluación de las poblaciones de las plantas útiles.

\section{Materiales y métodos}

El trabajo se realizó en el Valle de Tehuacán $\left(17^{\circ} 48^{\prime}\right.$ $\left.18^{\circ} 58^{\prime} \mathrm{N}, 97^{\circ} 03^{\prime}-97^{\circ} 43^{\prime} \mathrm{O}\right)$, ubicado en el noreste del estado de Oaxaca y sureste del estado de Puebla, México. La aridez de esta región es resultado de la sombra orográfica producida por la sierra Madre Oriental. La precipitación y la temperatura promedio anuales varían entre 400-500 mm y $18-22^{\circ} \mathrm{C}$, respectivamente (Valiente 1991). Algunos de los principales tipos de vegetación existentes en el valle son: 1), bosques de cactáceas columnares dominados por especies como: Cephalocereus columna-trajani, Escontria chiotilla, Neobuxbaumia tetetzo, Pachycereus weberi y Stenocereus stellatus; 2), mezquitales dominados por Prosopis laevigata y 3), selva baja caducifolia dominada por Ceiba parvifolia, Fouquieria formosa y varias especies de Bursera y Mimosa (Valiente-Banuet et al., 2000).

Las especies estudiadas tienen poblaciones en diferentes partes del valle, las cuales crecen en distintas condiciones ambientales y están sujetas a diversas formas de uso y manejo (Cuadro 1). Las poblaciones de S. stellatus son Zapotitlán y Tilapa. En la primera, el tipo de vegetación es matorral xerófilo; en la segunda, es selva baja caducifolia. Las prácticas de manejo en Tilapa no han sido evaluadas apropiadamente, pero son similares a las que se realizan actualmente en Zapotitlán (recolección). Las poblaciones de P. laevigata y C. tortuosa crecen en 2 terrazas aluviales del río Salado en Zapotitlán Salinas, Puebla (Terraza 1 y Terraza 2). El tipo de vegetación (mezquital) y las prácticas de manejo (recolección) en ambas terrazas son similares, pero difieren en las características del suelo y la estructura de la vegetación. La Terraza 1 tiene un suelo franco arenoso y una vegetación con fisonomía y estratificación vertical compleja, mientras que la Terraza 2 tiene un suelo franco arcilloso y una vegetación relativamente simple compuesta principalmente por el estrato arbóreo (LópezGalindo et al., 2003). Las poblaciones de A. marmorata 
Cuadro 1. Resumen de la información etnobotánica de las 4 especies estudiadas

\begin{tabular}{|c|c|c|c|c|}
\hline $\begin{array}{l}\text { Especie } \\
\text { (familia) }\end{array}$ & Nombres comunes & Usos & Manejo & Referencias \\
\hline $\begin{array}{l}\text { Stenocereus } \\
\text { stellatus } \\
\text { (Cactaceae) }\end{array}$ & $\begin{array}{l}\text { Xoconochtli (nahuatl), } \\
\text { Dichi (mixteco), Pitaya } \\
\text { (español) }\end{array}$ & $\begin{array}{c}\text { Forraje (tallos), alimento, } \\
\text { elaboración de bebidas } \\
\text { alcohólicas (frutos), leña (tallos } \\
\text { de plantas muertas), cerca, } \\
\text { control de suelos (plantas vivas) }\end{array}$ & $\begin{array}{c}\text { Poblaciones silvestres, } \\
\text { manejadas in situ y } \\
\text { cultivadas }\end{array}$ & $\begin{array}{c}\text { Casas et al., 2001; } \\
\text { Luna-Morales et al., } \\
2001\end{array}$ \\
\hline $\begin{array}{l}\text { Prosopis } \\
\text { laevigata } \\
\text { (Leguminosae) }\end{array}$ & Mezquite (español) & $\begin{array}{l}\text { Forraje, leña, material de } \\
\text { construcción (tallos), alimento } \\
\text { (frutos), medicinal (hojas y } \\
\text { flores), pegamento (resina de los } \\
\text { tallos) }\end{array}$ & $\begin{array}{c}\text { Poblaciones silvestres, } \\
\text { manejadas in situ } \\
\text { y algunas plantas } \\
\text { cultivadas en huertos }\end{array}$ & $\begin{array}{l}\text { Casas et al., 2001; } \\
\text { Paredes-Flores, } 2001\end{array}$ \\
\hline $\begin{array}{l}\text { Castela tortuosa } \\
\text { (Simaroubaceae) }\end{array}$ & $\begin{array}{c}\text { Chaparro amargoso } \\
\text { (español) }\end{array}$ & $\begin{array}{c}\text { Medicinal (corteza, ramas, } \\
\text { hojas, frutos) }\end{array}$ & Poblaciones silvestres & $\begin{array}{c}\text { Casas et al., 2001; } \\
\text { Paredes-Flores, } 2001\end{array}$ \\
\hline $\begin{array}{l}\text { Agave } \\
\text { marmorata } \\
\text { (Agavaceae) }\end{array}$ & Pichomel (español) & $\begin{array}{c}\text { Forraje, materiales de } \\
\text { construcción (hojas, escapos } \\
\text { florales), elaboración de bebidas } \\
\text { alcohólicas (pencas/tallos), leña, } \\
\text { ornamento religioso (escapos } \\
\text { florales), cerca, control de suelos } \\
\text { (plantas vivas) }\end{array}$ & $\begin{array}{l}\text { Poblaciones silvestres } \\
\text { bajo diferentes } \\
\text { condiciones de } \\
\text { explotación }\end{array}$ & $\begin{array}{l}\text { Casas et al., 2001; } \\
\text { Paredes-Flores, 2001; } \\
\text { Jiménez et al., } 2004\end{array}$ \\
\hline
\end{tabular}

son Zapotitlán y Los Reyes. El tipo de vegetación en ambas poblaciones es matorral xerófilo. La primera no está sujeta a actividades de explotación y/o extracción, mientras que la segunda es una fuente principal de extracción de aguamiel para elaborar bebidas alcohólicas.

El trabajo de campo para analizar los atributos demográficos de las 4 especies de plantas se realizó entre 2002 y 2004 (S. stellatus , 2002; P. laevigata y A. marmorata, 2003; C. tortuosa, 2004). En cada población se realizaron 3-10 transectos de $10 \times 30-60 \mathrm{~m}$. Los transectos son la forma más adecuada para muestrear las poblaciones de estas especies debido a su forma de crecimiento. El tamaño y el número de los transectos dependieron del tamaño de la población y las condiciones fisiográficas existentes en cada sitio. Los transectos se establecieron paralelamente, con una separación de al menos $10 \mathrm{~m}$, tratando de representar la mayor parte de la población. Todos los individuos encontrados en los transectos se marcaron y contaron, excepto los de $A$. marmorata que fueron contados en su totalidad en ambas poblaciones. La densidad de cada población se estimó como el número promedio de individuos por transecto y se comparó con una $\chi^{2}$ (Zar, 1988). Los individuos dentro de cada transecto se midieron con una pértiga o flexómetro para estimar su tamaño individual. En S. stellatus, se midió la altura de todas las ramas de cada individuo y el tamaño individual se calculó como la suma de la altura de todas las ramas.
En P. laevigata y C. tortuosa, se midió la altura $(h)$ y dos diámetros perpendiculares de la copa de cada individuo $\left(D_{1}, D_{2}\right)$. El tamaño individual de $P$. laevigata se calculó como el volumen de un cono $\left(V=\pi r^{2} h / 3\right)$, mientras que el de $C$. tortuosa se calculó como el volumen de un elipsoide $\left(V=1 / 6 \pi h\left(3 r^{2}+h^{2}\right)\right.$. Para calcular $r^{2}$ en ambas especies, cada uno de los diámetros perpendiculares se dividió primero entre 2 y después se multiplicaron entre sí. En A. marmorata, se midió el diámetro máximo de cada individuo.

Los individuos de cada especie fueron clasificados en 11-15 categorías de tamaño. Las categorías para S. stellatus fueron (en m): 1) < 0.05, 2) 0.05-0.1, 3) 0.1-0.5, 4) 0.5-1, 5) 1-2, 6) 2-3, 7) 3-4, 8) 4-5, 9) 5-7, 10) 7-9 у 11) > 9. En P. laevigata $\left(\mathrm{en} \mathrm{m}^{3}\right)$ : 1) < 0.1, 2) 0.1-0.5, 3) 0.5-1, 4) $1-5,5) 5-10,6) 10-20,7)$ 20-30, 8) 30-40, 9) 40-50, 10) $50-60,11) 60-70,12) 70-80,13) 80-90,14) 90-100$ у 15) $>100$. En C. tortuosa $\left(\mathrm{en} \mathrm{m}^{3}\right)$ : 1) < 0.01, 2) 0.01-0.05, 3) $0.05-0.1,4) 0.1-0.5,5) 0.5-1,6) 1-5,7)$ 5-10, 8) 10-20, 9) 20-30, 10) 30-40, 11) 40-50, 12) 50-60 y 13) > 60. En $A$. marmorata (en m): 1) <0.1, 2) 0.1-0.2, 3) 0.2-0.4, 4) 0.4$0.6,5)$ 0.6-0.8, 6) $0.8-1,7)$ 1-1.2, 8) 1.2-1.4, 9) 1.4-1.6, 10) $1.6-1.8$, 11) $1.8-2$ y 12) > 2. Las categorías de tamaño se definieron con base en el rango de los datos obtenidos en campo. Las estructuras poblacionales de cada especie fueron comparadas con una $\chi^{2}$ (Zar 1988).

La importancia relativa de la propagación vegetativa 
y la reproducción sexual se determinó sólo en $S$. stellatus y A. marmorata, considerando el origen de los individuos $<0.5 \mathrm{~m}$ y $<0.1 \mathrm{~m}$, respectivamente. Para cada individuo encontrado dentro de los transectos de la estructura poblacional se determinó si provenía de reproducción sexual o propagación vegetativa. Los individuos unidos a raíces (A. marmorata) y ramas caídas (S. stellatus), o con evidencia de uniones previas a plantas adultas fueron considerados derivados de propagación vegetativa. Estos criterios son relativamente confiables puesto que se ha observado que A. marmorata y $S$. stellatus solamente se propagan a través de meristemos ubicados en raíces y tallos, respectivamente $(\mathrm{H}$. Godínez, obs. pers.). Los individuos que no cumplían con estos criterios se consideraron derivados de reproducción sexual. Las diferencias en el número de individuos generados por reproducción sexual y los de propagación vegetativa entre las poblaciones de cada especie fueron analizadas con la prueba exacta de Fisher (Zar 1988).

Para determinar el número de frutos por planta, se realizaron censos cada 15 días de los individuos encontrados en los transectos de la estructura poblacional. Los censos de $S$. stellatus se realizaron en 2002, los de $P$. laevigata y A. marmorata en 2003, y los de C. tortuosa en 2004. Los censos se realizaron dependiendo de la forma de crecimiento de cada especie. En $S$. stellatus y A. marmorata se contó el número total de frutos por planta, mientras que en $P$. laevigata y C. tortuosa sólo se contó el número de frutos en una sección de la planta. En P. laevigata se contó el número de frutos por rama principal y el número total de frutos se estimó como el producto del número de frutos por rama por el número de ramas principales de la planta. En C. tortuosa se contó el número de frutos en una cuarta parte del volumen de la copa y el número total de frutos se estimó multiplicando por 4 el número de frutos encontrado. El número promedio de frutos por planta se comparó entre las poblaciones de cada especie con una $t$ de Student, previa transformación logarítmica de los datos, para cumplir con los criterios de normalidad y homogeneidad de varianza.

Para conocer el número de semillas por fruto se colectaron 20-73 frutos en cada una de las poblaciones de las especies. Las diferencias en el número promedio de semillas por fruto entre poblaciones se analizaron con una $t$ de Student (Zar 1988). Los datos se transformaron logarítmicamente para cumplir con los criterios de normalidad y homogeneidad de varianza.

La germinación se analizó mediante experimentos en laboratorio. Para cada especie, se colectaron 25-100 frutos por población para obtener semillas, las cuales fueron separadas por disección, desinfectadas con hipoclorito de sodio (30\%, $5 \mathrm{~min}$ ) y lavadas con agua destilada (5 min). La unidad experimental fue una caja de Petri o charola de plástico, con agrolita o papel filtro húmedo y 10-50 semillas. Para cada población se realizaron 3-10 repeticiones que se colocaron en condiciones ambientales de luz y temperatura (P. laevigata y $C$. tortuosa) o en una cámara ambiental $\left(25^{\circ} \mathrm{C}\right.$, luz fluorescente y $12 \mathrm{~h}$ de fotoperiodo; S. stellatus y A. marmorata). Las diferencias en los métodos entre las especies se debieron a diferencias en el tamaño de las semillas y las condiciones adecuadas para su germinación. Las repeticiones se revisaron diariamente para contar el número de semillas germinadas y mantener condiciones constantes de humedad. El criterio para considerar germinada una semilla fue la emergencia de la radícula. Para cada especie, se calculó la proporción promedio de semillas germinadas por población y se comparó con una $t$ de Student, previa transformación arcoseno de los datos (Zar 1988).

Para analizar la supervivencia se realizaron experimentos en campo, en los que las plántulas de cada una de las especies se colocaron en 2 condiciones ambientales: debajo de plantas perennes y en sitios desprovistos de vegetación. Las plántulas usadas en estos experimentos se obtuvieron colocando 100-500 semillas de cada especie, en recipientes de plástico con suelo de cada población. El suelo se regó a capacidad de campo y se revisó diariamente para agregar agua y mantener condiciones constantes de humedad. Los recipientes se colocaron en condiciones ambientales de luz y temperatura ( $P$. laevigata y C. tortuosa) o en una cámara ambiental $\left(25^{\circ} \mathrm{C}\right.$, luz fluorescente y $12 \mathrm{~h}$ de fotoperiodo; $S$. stellatus y $A$. marmorata).

Las plántulas emergidas se aclimataron gradualmente a condiciones de campo por un periodo de 7-90 días, mediante el incremento en la exposición a la radiación solar y la disminución de la humedad del suelo. Después de este tiempo, las plántulas se llevaron al campo, donde se colocaron debajo de Mimosa luisana (plántulas de $S$. stellatus y A. marmorata), Prosopis laevigata (plántulas de P. laevigata) y Castela tortuosa (plántulas de C. tortuosa). Estas especies fueron seleccionadas debido a que son las más abundantes en las poblaciones estudiadas. La unidad experimental fue una charola de plástico con suelo del sitio y 10-200 plántulas. En cada población, el número de repeticiones en cada condición ambiental fue de 2-8. Las repeticiones debajo de plantas perennes se colocaron en la base de plantas de tamaño relativamente similar. Las plántulas se contaron cada 15-30 días y los datos se analizaron con la prueba de Peto y Peto (Pyke y Thompson, 1980), para determinar si existían diferencias en el número de plántulas supervivientes debajo de plantas perennes y en el de los sitios desprovistos de vegetación.

\section{Resultados}

La densidad de las especies varió desde 0.04 hasta 0.12 
ind $/ \mathrm{m}^{2}$, aunque no hubo diferencias significativas entre ninguna de las poblaciones, excepto en P. laevigata. En esta especie, la densidad en la Terraza 1 fue mayor que en la Terraza 2 (Cuadro 2). La densidad de $A$. marmorata no se comparó entre poblaciones, debido a que se contaron todos los individuos existentes en cada una de ellas.

La estructura poblacional de S. stellatus $\left(\chi^{2}=11.5\right.$, g. $1.10, \mathrm{p}=0.32)$ y P. laevigata $\left(\chi^{2}=12.2\right.$, g. $1.14, \mathrm{p}$ $=0.59$ ) no difirió entre sus poblaciones (Fig. 1a, b). En ambas especies, la proporción de individuos de tamaño pequeño ( $<0.1 \mathrm{~m}$ en $S$. stellatus $;<1 \mathrm{~m}^{3}$ en $P$. laevigata) y grande ( $>4 \mathrm{~m}$ en $S$. stellatus; $>50 \mathrm{~m}^{3}$ en $P$. laevigata) fue de $5-8 \%$ y $14-20 \%$, respectivamente. La proporción de individuos de tamaño intermedio (0.1-4 m en S. stellatus; 1-50 $\mathrm{m}^{3}$ en $P$. laevigata) fue de $72-81 \%$. En C. tortuosa $\left(\chi^{2}=79.9\right.$, g. $\left.1.12, \mathrm{p}<0.00001\right)$ y A. marmorata $\left(\chi^{2}=20.7\right.$, g. 1. $11, \mathrm{p}=0.04)$, si hubo diferencias significativas en la estructura de sus poblaciones (Fig. 1c, d). Los individuos de $C$. tortuosa $<1 \mathrm{~m}^{3}$ tuvieron una mayor proporción relativa en la Terraza $2(52 \%)$ que en la Terraza $1(6 \%)$. De manera similar, la proporción relativa de los individuos de A. marmorata < $0.4 \mathrm{~m}$ fue mayor en Zapotitlán (52\%) que en Los Reyes (26\%).

La propagación vegetativa fue el modo de reproducción más común en todas las poblaciones de S. stellatus y $A$. marmorata (Fig. 2a, b). Del total de individuos muestreados, el $80-93 \%$ de $S$. stellatus provenía de ramas postradas en el suelo, mientras que el $75-100 \%$ de $A$. marmorata se encontraron asociados a las raíces de plantas adultas. En ambas especies, las plantas derivadas de semilla fueron escasas (7-25\%) y se encontraron asociadas a árboles y arbustos perennes como Prosopis laevigata y Mimosa luisana. No se encontró ningún individuo de $A$. marmorata derivado de semilla en la población de Los Reyes (Fig. 2b).

El número de frutos por planta varió desde 5 en $S$. stellatus hasta más de 350 en P. laevigata (Cuadro 2). En todas las especies se encontraron diferencias significativas entre las poblaciones, por lo que la producción individual de frutos varió dependiendo de la población. El número de semillas por fruto varió desde 1 en C. tortuosa hasta más de 1000 en S. stellatus (Cuadro 2). No se encontraron diferencias en el número de semillas por fruto entre las poblaciones de cada especie, excepto en $P$. laevigata. En esta especie, las plantas de la Terraza 2 produjeron un mayor número de semillas por fruto que las plantas de la Terraza 1. El número de frutos por planta y el número de semillas por fruto de $A$. marmorata no se comparó entre sus poblaciones, debido a que los individuos de Los Reyes no se reprodujeron sexualmente (Cuadro 2).

La germinación varió dependiendo de la especie de planta útil (Cuadro 2). Las semillas de todas las especies germinaron en los primeros 4 días (Fig. 3a-d). La especie A. marmorata presentó la mayor proporción promedio

Cuadro 2. Densidad, producción de frutos y semillas, germinación y supervivencia de plántulas de 4 especies de plantas útiles en el Valle de Tehuacán, México

\begin{tabular}{|c|c|c|c|c|c|c|c|}
\hline \multirow[t]{2}{*}{ Especie } & \multirow[t]{2}{*}{ Población $^{\#}$} & \multirow[t]{2}{*}{ Densidad $^{\#}\left(\right.$ ind $\left./ m^{2}\right)$} & \multirow[t]{2}{*}{ Frutos/planta ${ }^{\#}$} & \multirow[t]{2}{*}{ Semillas/fruto } & \multirow{2}{*}{$\begin{array}{c}\text { Germinación }^{\#} \\
\text { (Proporción) }\end{array}$} & \multicolumn{2}{|c|}{ Supervivencia (ln) } \\
\hline & & & & & & $\begin{array}{c}\text { Sitios sin } \\
\text { vegetación }\end{array}$ & $\begin{array}{c}\text { Debajo de } \\
\text { plantas }\end{array}$ \\
\hline \multirow{2}{*}{$\begin{array}{l}\text { Stenocereus } \\
\text { stellatus }\end{array}$} & Zapotitlán & $0.073 \pm 0.009^{\mathrm{a}}$ & $18.5 \pm 4.3^{\mathrm{a}}$ & $1028.8 \pm 54.9^{\mathrm{a}}$ & $0.43 \pm 0.09^{\mathrm{a}}$ & $1.4^{\mathrm{c}}$ & $3.1^{\mathrm{a}}$ \\
\hline & Tilapa & $0.074 \pm 0.015^{\mathrm{a}}$ & $4.9 \pm 0.7^{\mathrm{b}}$ & $1090 \pm 100.1^{\mathrm{a}}$ & $0.28 \pm 0.07^{\mathrm{a}}$ & $0.2^{\mathrm{d}}$ & $2.7^{\mathrm{b}}$ \\
\hline \multirow{2}{*}{$\begin{array}{l}\text { Prosopis } \\
\text { laevigata }\end{array}$} & Terraza 1 & $0.036 \pm 0.005^{\mathrm{a}}$ & $221.2 \pm 33.4^{\mathrm{a}}$ & $14.1 \pm 0.3^{\mathrm{a}}$ & $0.37 \pm 0.15^{\mathrm{a}}$ & 0 & $0.18^{c}$ \\
\hline & Terraza 2 & $0.121 \pm 0.01^{\mathrm{b}}$ & $351.5 \pm 32.5^{\mathrm{b}}$ & $20 \pm 0.3^{b}$ & $0.34 \pm 0.15^{\mathrm{a}}$ & $0.87^{\mathrm{a}}$ & $0.34^{\mathrm{b}}$ \\
\hline \multirow{2}{*}{$\begin{array}{l}\text { Castela } \\
\text { tortuosa }\end{array}$} & Terraza 1 & $0.097 \pm 0.014^{\mathrm{a}}$ & $157.9 \pm 23.4^{\mathrm{a}}$ & 1 & $0.5 \pm 0.2^{\mathrm{a}}$ & 0 & 0 \\
\hline & Terraza 2 & $0.062 \pm 0.0002^{\mathrm{a}}$ & $59.5 \pm 15.3^{b}$ & 1 & $0.8 \pm 0.1^{\mathrm{a}}$ & 0 & 0 \\
\hline \multirow{2}{*}{$\begin{array}{l}\text { Agave } \\
\text { marmorata }\end{array}$} & Zapotitlán & 0.09 & $230 \dagger$ & $224 \pm 4.8$ & 1.0 & $0^{\mathrm{a}}$ & $1.1^{\mathrm{b}}$ \\
\hline & Los Reyes & 0.11 & 0 & 0 & 0 & 0 & 0 \\
\hline
\end{tabular}

\footnotetext{
\# Media \pm error estándar

${ }^{\S}$ La densidad se determinó de manera absoluta y los individuos de Los Reyes no se reprodujeron sexualmente, por lo que no se realizaron comparaciones entre las poblaciones.

$\dagger$ No se tiene una medida de la variación individual .

Para cada atributo demográfico, las letras diferentes señalan diferencias significativas entre las poblaciones de cada especie.
} 
a)

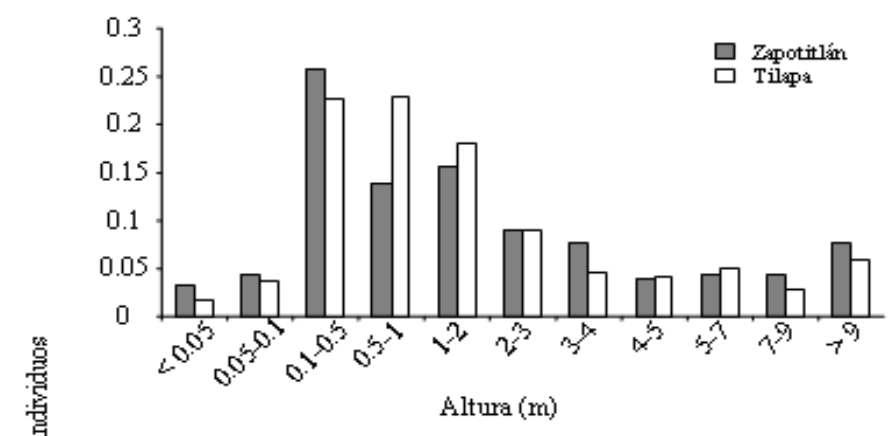

c)

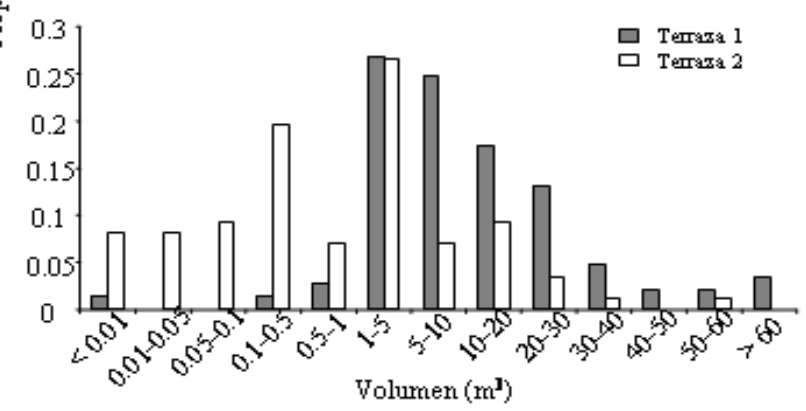

b)

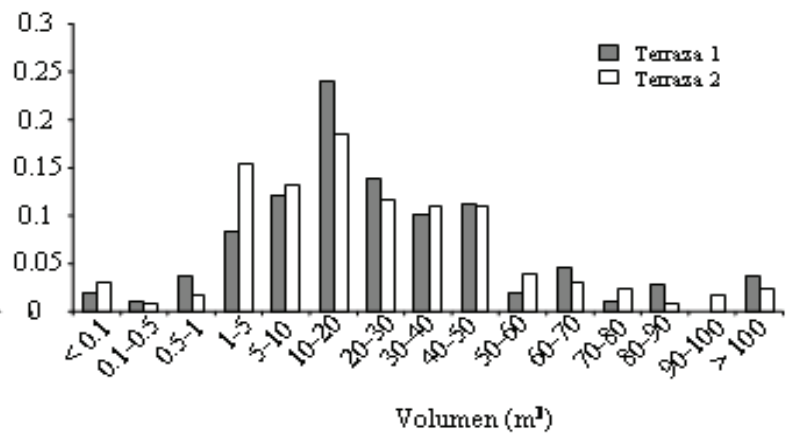

d)

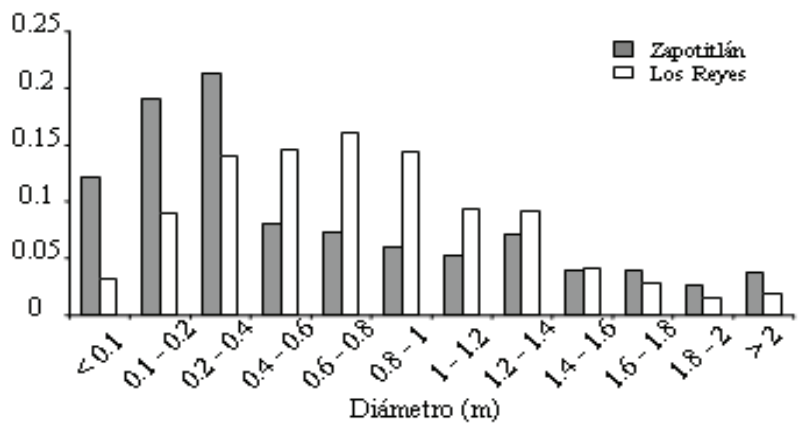

Figura 1. Estructura poblacional de Stenocereus stellatus a), Prosopis laevigata b), Castela tortuosa c) y Agave marmorata d), en el Valle de Tehuacán, México.

de germinación (1.0), seguida de $C$. tortuosa $(0.5-0.8)$ y por último, P. laevigata (0.28-0.43) y S. stellatus (0.34$0.37)$. No se encontraron diferencias significativas entre las poblaciones de las 4 especies.

La supervivencia de las plántulas fue diferente dependiendo de la especie de planta y las condiciones ambientales (Cuadro 2). Las plántulas de S. stellatus y A. marmorata permanecieron vivas más de 160 y 300 días, respectivamente. La supervivencia de ambas especies en todas las poblaciones fue mayor debajo de plantas perennes que en sitios desprovistos de vegetación. Las plántulas de $P$. laevigata sobrevivieron alrededor de 80 días. La supervivencia en la Terraza 1 fue mayor debajo de plantas perennes, mientras que en la Terraza 2 fue mayor en sitios desprovistos de vegetación. Las plántulas de C. tortuosa sobrevivieron menos de 17 días en todos los tratamientos, por lo que no fue posible realizar ninguna pruebas estadísticas (Fig. 4a-d).

\section{Discusión}

En este trabajo se evaluaron distintos atributos demográficos para determinar el estado de las poblaciones de 4 especies de plantas útiles. Esta información es importante dado que los productos obtenidos de estas plantas contribuyen a la economía de los pobladores locales (Pérez-Negrón y Casas, 2007). Las poblaciones de todas las especies estudiadas presentan características que podrían limitar su capacidad de regeneración, afectando el uso que los habitantes pueden hacer de ellas. Todas las poblaciones presentaron densidades variables y la proporción de individuos pequeños y grandes fue baja. Estas características deben ser consideradas en el manejo y aprovechamiento de estas plantas, ya que se ha sugerido que las poblaciones compuestas por pocos individuos pequeños y grandes potencialmente pueden decrecer (Elzinga et al., 2001). Estudios demográficos de plantas 


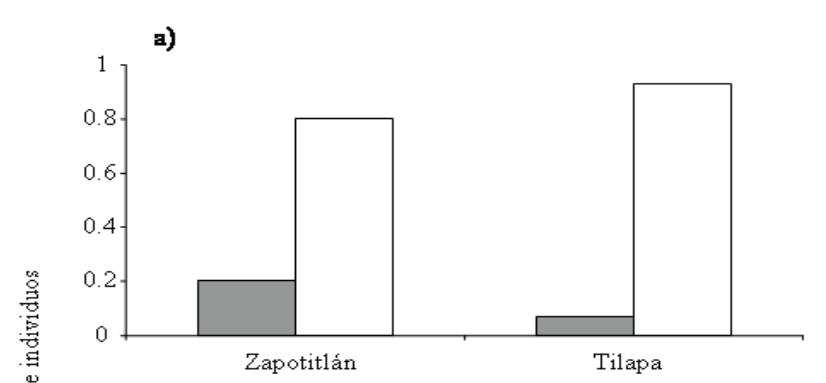

b)

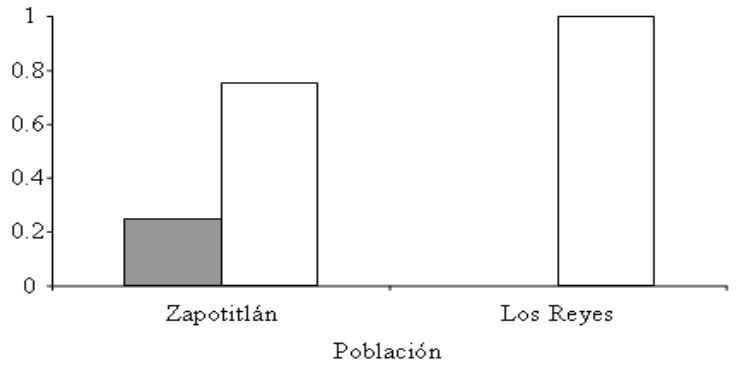

Figura 2. Proporción de individuos de Stenocereus stellatus (a) y Agave marmorata (b) derivados de reproducción sexual (barras llenas) y propagación vegetativa (barras vacías), en el Valle de Tehuacán, México.

con formas de crecimiento similares han sugerido que la supervivencia de las plántulas y las plantas adultas afecta el crecimiento de las poblaciones (Godínez-Álvarez et al., 2003; Jiménez-Lobato y Valverde, 2006).

Los resultados muestran que la estructura poblacional de C. tortuosa difiere entre los sitios estudiados. Estos sitios varían en el tipo de suelo y la estructura de la vegetación. El sitio Terraza 1 tiene un suelo franco arenoso y una vegetación con una estratificación vertical compleja, mientras que el sitio Terraza 2 tiene un suelo franco arcilloso y una vegetación compuesta sólo por un estrato arbóreo (López-Galindo et al., 2003). Algunos autores (Medina, 2000; Ruedas et al., 2006) han sugerido que la textura del suelo puede determinar su capacidad de retención de agua y contenido de nutrientes, afectando la germinación de las semillas y la supervivencia de las plántulas. Los suelos con texturas arenosas tiene una menor capacidad de retención de agua y nutrientes que los suelos con texturas arcillosas (Medina, 2000; Ruedas et al., 2006). Las diferencias en el tipo de suelo entre las terrazas podrían explicar las diferencias en las estructuras poblacionales. Sin embargo, es importante considerar que otros factores físicos (altitud, precipitación) y biológicos (interacciones biológicas) también pueden afectar las tasas de natalidad y mortalidad individuales, por lo que es necesario realizar otros trabajos para conocer la importancia de todos estos factores en la estructuración de las poblaciones. En el caso de $A$. marmorata, el uso por parte de los habitanteslocales es un factor que aparentemente puede afectar su estructura poblacional. El número de plántulas de $A$. marmorata encontrado en la población sujeta a explotación (Los Reyes) fue menor que el número de plántulas encontrado en la población que no está sujeta a extracción/explotación (Zapotitlán).

La producción de frutos fue diferente entre las especies y entre las poblaciones de cada especie. Las diferencias interespecíficas son un resultado esperado si consideramos que las plantas estudiadas pertenecen a familias botánicas diferentes, con características morfológicas y fisiológicas propias. Las plantas de todas las especies, excepto $A$. marmorata, siempre produjeron un mayor número de frutos en alguna de las poblaciones. Estas diferencias sugieren que las poblaciones con la mayor reproducción individual podrían ser las más adecuadas para la extracción de frutos. No obstante lo anterior, es importante recordar que la producción de frutos se analizó sólo durante un año, por lo que para tener una idea más precisa de la variación existente entre las poblaciones es necesario realizar estudios de mayor duración.

El uso de las estructuras reproductivas (flores, frutos y semillas) por los que habitan en la localidad es un factor que puede afectar el estado en el que se encuentran las poblaciones y su capacidad de regeneración. En este sentido, la falta de producción de frutos y semillas de A. marmorata en la población de Los Reyes podría deberse a que el escapo floral es cortado y utilizado para la construcción de nidos de canarios, lo cual impide la reproducción sexual (Jiménez et al., 2004). Es importante recordar, sin embargo, que en este trabajo sólo se analizó una población durante un año, por lo que es necesario realizar otros estudios sobre el posible efecto de la utilización de las estructuras reproductivas y la variación interanual de la reproducción. El efecto negativo del uso de ciertas estructuras reproductivas también ha sido registrado para otras especies útiles como Bertholletia excelsa (Peres et al., 2003), Thrinax radiata y Cocothrinax readii (Olmstead y Álvarez Buylla, 1995).

La supervivencia de las plántulas de todas las especies fue relativamente baja y dependió fuertemente de las condiciones microambientales. En algunas especies como S. stellatus y A. marmorata, los sitios ubicados debajo de plantas perennes fueron los que presentaron la mayor supervivencia de plántulas, mientras que en $P$. laevigata la mayor supervivencia ocurrió tanto en espacios abiertos como debajo de arbustos. Este patrón de supervivencia es similar al reportado para otras especies de plantas de zonas áridas con distintas formas de crecimiento (Flores y Briones, 2001; Godínez-Álvarez et al., 2003).

$\mathrm{El}$ requerimiento de condiciones microambientales 
a)

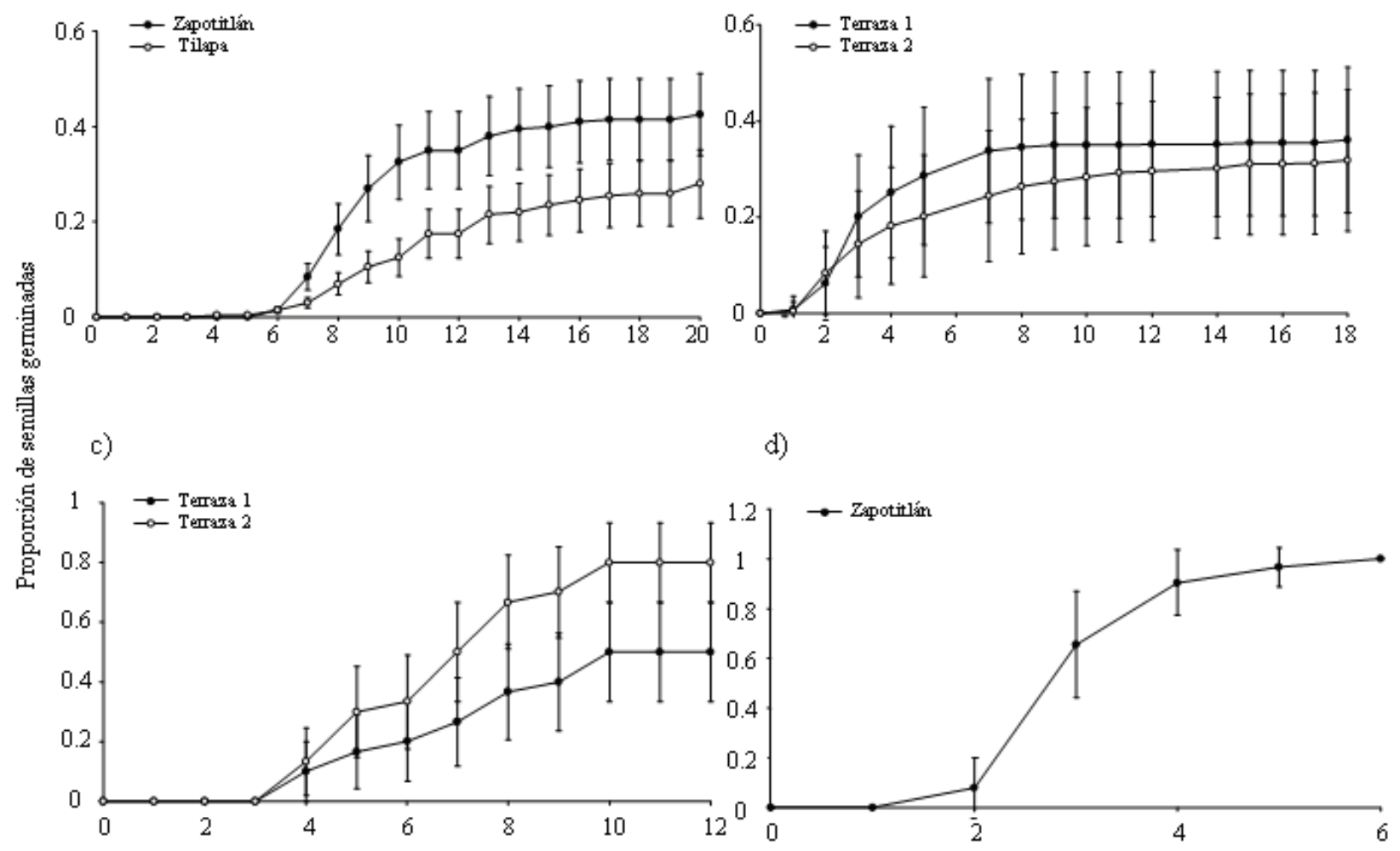

Tiempo (dias)

Figura 3. Proporción de semillas germinadas ( \pm error estándar) de Stenocereus stellatus a), Prosopis laevigata b), Castela tortuosa c) y Agave marmorata d), en condiciones controladas. Los círculos llenos y vacíos indican las poblaciones en donde fueron colectadas las semillas.

específicas para la supervivencia de las plántulas determina que el establecimiento por semilla sea un evento poco frecuente. Esta situación debe ser considerada en el manejo de estas plantas útiles, debido a que la reproducción sexual en algunas especies como $C$. tortuosa y $P$. laevigata es el único mecanismo existente para el reclutamiento de nuevos individuos en las poblaciones. Otras especies como S. stellatus y A. marmorata tienen otra forma de reproducción, como la propagación vegetativa, la cual puede contribuir al mantenimiento de sus poblaciones. Los resultados sugieren que la propagación vegetativa es el mecanismo más frecuente de reproducción en estas especies. La propagación vegetativa de S. stellatus se interpreta como un mecanismo contra la alta incertidumbre de las lluvias, la cual puede afectar el establecimiento de las plántulas (Godínez-Álvarez et al., 2005).

Este trabajo muestra que la evaluación de los atributos demográficos proporciona información que permite determinar el estado en el que se encuentran las poblaciones y reflexionar respecto de los factores físicos y biológicos que podrían limitar su uso. Recientemente se ha sugerido que la estimación de la densidad de las especies útiles, junto con la estimación de las tasas de extracción de los recursos por los pobladores, es un método rápido que permitiría obtener información para la elaboración de planes de manejo de estas plantas (Pérez-Negrón y Casas, 2007). Nosotros creemos que la estimación de la densidad es insuficiente para entender el estado actual de las poblaciones y los posibles impactos de la extracción. Distintas poblaciones de plantas de la misma especie pueden presentar densidades similares, pero estar conformadas por individuos de distintas categorías de tamaño (p. ej., altas proporciones de plantas seniles o no reproductivas). Estas diferencias afectan la capacidad de regeneración de 
a)
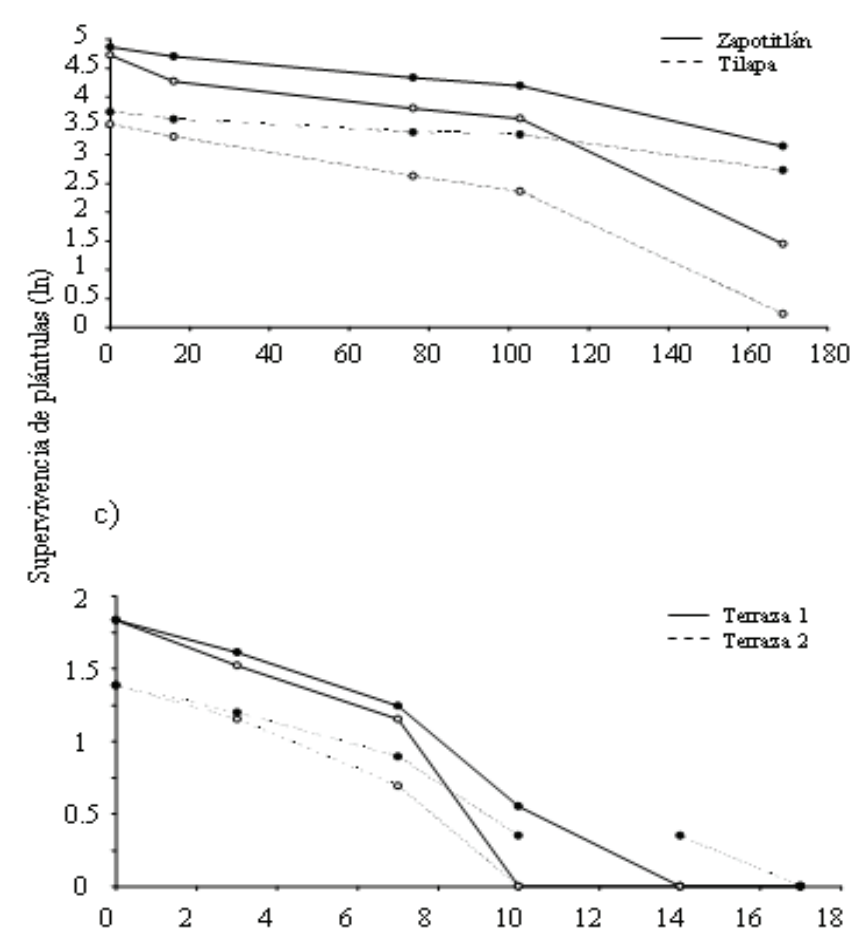

b)

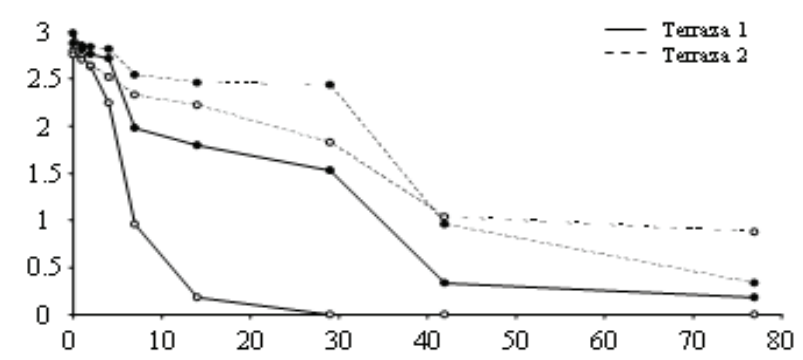

d)

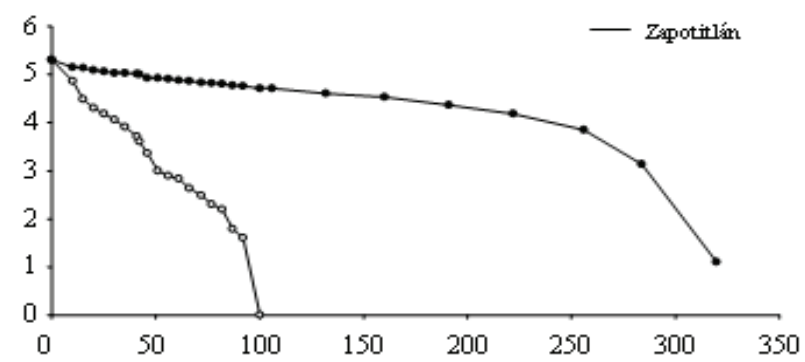

Tiempo (dias)

Figura 4. Supervivencia de plántulas de Stenocereus stellatus a), Prosopis laevigata b), Castela tortuosa c) y Agave marmorata d), en distintas poblaciones del Valle de Tehuacán, México. Los círculos vacíos y llenos se refieren a las plántulas que crecen en espacios desprovistos de vegetación y debajo de arbustos, respectivamente.

las poblaciones (Elzinga et al., 2001; Silvertown, 1987).

Los estudios demográficos de las plantas útiles con modelos matriciales son otra alternativa para obtener información para el manejo de estas plantas, ya que permiten determinar los factores que limitan el crecimiento poblacional y los niveles adecuados de extracción de los recursos (Martínez-Ballesté et al., 2005; Olmstead y Álvarez-Buylla, 1995). Este tipo de estudios, sin embargo, no siempre describen adecuadamente la dinámica poblacional de las plantas longevas de regiones áridas y semiáridas. Esta limitante es especialmente importante en el Valle de Tehuacán, donde existen más de 900 especies de plantas útiles (Pérez-Negrón y Casas, 2007). Considerando lo anterior, nosotros creemos que una tercera alternativa para determinar la condición de las especies útiles podría ser la evaluación de los atributos demográficos de las poblaciones. La medición de todos o algunos de estos atributos (p. ej. número de plántulas, número de plantas reproductivas, número de frutos por planta, etc.) cada cierto número de años permitiría dar seguimiento a los cambios poblacionales.

\section{Agradecimientos}

Este trabajo fue financiado por la International Foundation for Science (IFS), proyecto D/3202-1, y el Programa de Apoyo a Proyectos de Investigación e Innovación Tecnológica (PAPIIT), proyectos IN213402-2 e IX247904.

\section{Literatura citada}

Avendaño, A., A. Casas, P. Dávila y R. Lira. 2006. Use forms, management and commercialization of "pochote" Ceiba aesculifolia (H.B. and K.) Britten and Baker f. subsp. parvifolia (Rose) P.E. Gibbs and Semir (Bombacaceae) in the Tehuacán Valley, Central Mexico. Journal of Arid 
Environments 67:15-35.

Blanckaert, I., R. L. Swennen, M. Paredes, R. Rosas y R. Lira. 2004. Floristic composition, plant uses and management practices in homegardens of San Rafael Coxcatlán, Valley of Tehuacán-Cuicatlán, Mexico. Journal of Arid Environments 57:179-202.

Camargo-Ricalde, S. L., S. S. Dhillion y V. García-García. 2004. Phenology, and seed production and germination of seven endemic Mimosa species (Fabaceae-Mimosoideae) of the Tehuacán-Cuicatlán Valley, Mexico. Journal of Arid Environments 58:423-437.

Carmona, A. y A. Casas. 2005. Management, phenotypic patterns and domestication of Polaskia chichipe (Cactaceae) in the Tehuacán Valley, central Mexico. Journal of Arid Environments 60:115-132.

Casas, A., B. Pickersgill, J. Caballero y A. Valiente-Banuet. 1997. Ethnobotany and domestication in xoconochtli, Stenocereus stellatus (Cacataceae), in the Tehuacan Valley and La Mixteca Baja, Mexico. Economic Botany 51:279-292.

Casas, A., J. Caballero, A. Valiente-Banuet, J. A. Soriano y P. Dávila. 1999a. Morphological variation and the process of domestication of Stenocereus stellatus (Cactaceae) in central Mexico. American Journal of Botany 86:522-533.

Casas, A., A. Valiente-Banuet, A. Rojas-Martínez y P. Dávila. 1999b. Reproductive biology and the process of domestication of the columnar cactus Stenocereus stellatus in central Mexico. American Journal of Botany 86:534-542.

Casas A., A. Valiente-Banuet, J. L. Viveros, J. Caballero, L. Cortez, P. Dávila, R. Lira, e I. Rodríguez. 2001. Plant resources of the Tehucán-Cuicatlán Valley, México. Economic Botany 55:129-166.

Cruz, M. y A. Casas. 2002. Morphological variation and reproductive biology of Polaskia chende (Cactaceae) under domestication in Central Mexico. Journal of Arid Environments 51:561-576.

Dávila, P., M. C. Arizmendi, A. Valiente-Banuet, J. L. Villaseñor, A. Casas y R. Lira. 2002. Biological diversity in the Tehuacán-Cuicatlán Valley, Mexico. Biodiversity and Conservation 11:421-442.

Elzinga, C. L., D. W. Salzer, J. W. Willoughby y J. P. Gibbs. 2001. Monitoring plant and animal populations. Blackwell Science, London. $360 \mathrm{p}$.

Flores, J. y O. Briones. 2001. Plant life-form and germination in a Mexican inter-tropical desert: effects of soil water potential and temperature. Journal of Arid Environments 47:485-497.

Godínez-Alvarez, H., T. Valverde y P. Ortega-Baes. 2003. Demographic trends in the Cactaceae. The Botanical Review 69:173-203.

Godínez-Alvarez, H., L. Ríos-Casanova y F. Pérez. 2005. Characteristics of seedling establishment of Stenocereus stellatus (Cactaceae) in the Tehuacán Valley, Mexico. The Southwestern Naturalist 50:375-380.

Jiménez, V. M., R. Lira, H. Godínez-Alvarez y J. Caballero. 2004. Evaluación del efecto de las formas de aprovechamiento sobre la dinámica poblacional de Agave marmorata Roezl. (Agavaceae) en el Valle de Tehuacan-Cuicatlán: un estudio demográfico. Simposio Aplicaciones Ecológicas y Etnobotánicas en la Evaluación de Sostenibilidad:
Perspectivas para el Manejo de los Recursos Naturales. XVI Congreso Mexicano de Botánica. Sociedad Botánica de México, Oaxaca, Oax.

Jiménez-Lobato, V. y T. Valverde. 2006. Population dyanmics of the shrub Acacia bilimekii in a semi-desert region in central Mexico. Journal of Arid Environments 65:29-45.

López-Galindo, F., D. Muñoz-Iniestra, M. Hernández-Moreno, A. Soler-Aburto, M. C. Castillo-López e I. Hernández-Arzate. 2003. Análisis integral de la toposecuencia y su influencia en la distribución de la vegetación y la degradación del suelo en la subcuenca de Zapotitlán Salinas, Puebla. Boletín de la Sociedad Geológica Mexicana 56:19-41.

Luna-Morales, C., J. R. Aguirre-Rivera y C. B. Peña-Valdivia. 2001. Cultivares tradicionales mixtecos de Stenocereus pruinosus y $S$. stellatus (Cactaceae). Anales del Instituto de Biología, Universidad Nacional Autónoma de México, Serie Botánica 72:131-155.

Martínez-Ballesté, A., C. Martorell, M. Martínez-Ramos y J. Caballero. 2005. Applying retrospective demographic models to assess sustainable use: the Maya management of xa'an palms. Ecology and Society http://www.ecologyandsociety. org/vol11/iss2/art27/

Medina, S. J., 2000. Determinación del vigor y el estado reproductivo de Stenocereus stellatus (Cactaceae) a lo largo de una cronosecuencia edáfica en un abanico aluvial en Coxcatlán, Valle de Tehuacán. Tesis, Facultad de Estudios Superiores Iztacala, UNAM, México, D. F. 48 p.

Mendoza, O. M., 2006. Características demográficas de Castela tortuosa Liebm., en dos terrazas aluviales con distinto grado de deterioro en el Valle de Zapotitlán de las Salinas, Puebla. Tesis, Facultad de Estudios Superiores Iztacala, UNAM, México, D. F. 42 p.

Olmsted, I. y E. R. Álvarez-Buylla. 1995. Sustentable harvesting of tropical trees: demograghy and matrix models of two palm species in México. Ecological Applications 5:484-500.

Otero-Arnaiz, A., A. Casas, C. Bartolo, E. Pérez-Negrón y A. Valiente- Banuet. 2003. Evolution of Polaskia chichipe (Cactaceae) under domestication in the Tehuacán Valley, central Mexico: reproductive biology. American Journal of Botany 90:593-602.

Paredes-Flores, M., 2001.Contribución al estudio etnobotánico de Zapotitlán de las Salinas, Puebla. Tesis, Facultad de Estudios Superiores Iztacala, UNAM, México, D. F. 104 p.

Peres, C. A., C. Baider, P. A. Zuidema, L. H. O. Wadt, K. A. Kainer, D. A. P. Gomes-Silva, R. P. Salomao, L. L. Simoes, E. R. N. Franciosi, F. C. Valverde, R. Gribel, G. H. Shepard Jr., M. Kanashiro, P. Coventry, D. W. Yu, A. R. Watkinson y R. P. Freckleton. 2003. Demographic threats to the sustainability of Brazil nut exploitation. Science 302:2112-2114.

Pérez, V. A., 2004. Aspectos demográficos de dos poblaciones de Stenocereus stellatus, una cactácea endémica del centro de México. Tesis, Facultad de Estudios Superiores Iztacala, UNAM, México, D. F. 38 p.

Pérez-Negrón, E. y A. Casas. 2007. Use, extraction rates and spatial availability of plant resources in the TehuacánCuicatlán Valley, Mexico: The case of Santiago Quiotepec, Oaxaca. Journal of Arid Environments 70:356-379.

Pyke, D. y J. N. Thompson. 1980. Statistical analysis of survival 
and removal rate experiments. Ecology 67:240-245.

Roldán, M. P. 2004. Patrones demográficos de Prosopis laevigata en un ambiente fragmentado del Valle de Zapotitlán de las Salinas, Puebla. Tesis, Facultad de Estudios Superiores Iztacala, UNAM, México, D. F. 40 p.

Ruedas, M., T. Valverde y J. A. Zavala-Hurtado. 2006. Analysis of the factors that affect the distribution and abundance of three Neobuxbaumia species (Cactaceae) that differ in their degree of rarity. Acta Oecologica 29:155-164.

Silvertown, J. W. 1987. Introduction to plant population ecology.
Longman Scientific \& Technical, Essex, England. 229 p.

Valiente, B. L. 1991. Patrones de precipitación en el valle semiárido de Tehuacán, Puebla, México. Tesis, Facultad de Ciencias, UNAM, México, D. F. 61 p.

Valiente-Banuet, A., A. Casas, A. Alcántara, P. Dávila, N. FloresHernández, M. C. Arizmendi, J. L. Villaseñor, J. Ortega y J. A. Soriano. 2000. La vegetación del Valle de Tehuacán. Boletín de la Sociedad Botánica de México 67:25-74.

Zar, J. H. 1988. Biostatistical analysis. Prentice Hall, Englewood Cliffs, New Jersey. 718 p. 